\title{
Development of a Parkinson's disease specific falls questionnaire
}

\author{
Dale M. Harris ${ }^{1,2^{*}}$, Rachel L. Duckham,3,4, Robin M. Daly ', Gavin Abbott ${ }^{1}$, Liam Johnson ${ }^{5,6}$, \\ Timo Rantalainen ${ }^{1,7}$ and Wei-Peng Teo ${ }^{1,8}$
}

\begin{abstract}
Background: Falls are a major health burden for older adults with Parkinson's disease (PD), but there is currently no reliable questionnaire to capture the circumstances and consequences of falls in older adults with PD. This study aimed to develop a PD-specific falls questionnaire and to evaluate its test-retest reliability in older adults with PD.

Methods: A novel PD-specific falls questionnaire (PDF-Q) was developed in two modes (online and paper-based version) and used to assess falls and near-falls events over the past 12-months. Questions were agreed upon by an expert group, with the domains based on previous falls-related questionnaires. The questions included the number and circumstances (activities, location and direction) of falls and near-falls, and consequences (injuries and medical treatment) of falls. The PDF-Q was distributed to 46 older adults with PD (online $n=30$, paper $n=16$ ), who completed the questionnaire twice, 4 weeks apart. Kappa $(\mathrm{K})$ statistics were used to establish test-retest reliability of the questionnaire items.

Results: Pooled results from both questionnaires for all participants were used to assess the overall test-retest reliability of the questionnaire. Questions assessing the number of falls $(k=0.41)$ and the number of near-falls $(k=0.51)$ in the previous 12-months demonstrated weak agreement, while questions on the location of falls $(k=0.89)$ and near-falls $(k=1.0)$ demonstrated strong to almost perfect agreement. Questions on the number of indoor $(k=0.86)$ and outdoor $(k=0.75)$ falls demonstrated moderate to strong agreement, though questions related to the number of indoor ( $k=0.47)$ and outdoor ( $k=0.56)$ near-falls demonstrated weak agreement. Moderate to strong agreement scores were observed for the most recent fall and near-fall in terms of the direction (indoor fall $k=0.80$; outdoor fall $K=0.81$; near-fall $K=0.54$ ), activity (indoor fall $K=0.70$; outdoor fall $K=0.82$; near-fall $K=0.65$ ) and cause (indoor fall $K=0.75$; outdoor fall $K=0.62$; near-fall $K=0.56$ ).
\end{abstract}

Conclusions: The new PDF-Q developed in this study was found to be reliable for capturing the circumstances and consequences of recent falls and near-falls in older adults with PD.

Keywords: Parkinson's disease, Falls, Near-falls, Reliability, Questionnaire

\section{Background}

Falls are a major health burden for both healthy [1-3] and neurologically impaired [4] community-dwelling older adults as they are often associated with a loss of

\footnotetext{
*Correspondence: dale.harris36@outlook.com

${ }^{1}$ Institute for Physical Activity and Nutrition (IPAN), School of Exercise

and Nutrition Sciences, Deakin University, Geelong, Australia

Full list of author information is available at the end of the article
}

independence, poorer quality of life and even mortality $[5,6]$. It is estimated that between 28 to $36 \%$ of healthy community-dwelling individuals aged $\geq 65$ years fall each year $[7,8]$, but this incidence is markedly higher in older adults with Parkinson's disease (PD) whereby 35 to $90 \%$ are reported to fall at least once per year [9]. Although falls in healthy community-dwelling populations has been extensively explored [10-12], there is less research 
into the circumstances and consequences of falls for older adults with PD which may differ due to known risk factors that can adversely affect balance and posture, particularly for those with the postural instability and gait disturbance Parkinsonian subtype [13, 14]. Some of the key risk factors include axial rigidity causing poor leaning balance, cognitive impairments, bradykinetic responses to change-in-support strategies, hypokinetic gait and smaller foot clearance upon take-off $[6,15,16]$. Understanding the specific nature of the circumstances and consequences of falls experienced by older adults with PD is therefore important to better inform future falls prevention interventions and to mitigate falls risk factors.

To our knowledge, there are no reliable PD-specific falls questionnaires. To date, most of the previous research examining the circumstances and consequences of falls in older adults with PD have utilised either semistructured interviews or self-report falls diaries [17-24]. While these methods are the preferred choice when collecting falls information $[9,25]$, they can be costly, timeconsuming, have high attrition rates, and often require continued use of resources (e.g., for data entry and checking, and follow-up phone calls) [25]. In this respect, retrospective analysis using questionnaires to capture falls information may represent a viable alternative, but there are potential limitations such as recall biases [26].

In community-dwelling older adults, questionnaires have been used to assess falls risk factors and falls circumstances [27-30], including one using an online questionnaire to target frail older adults [31], yet the test-retest reliability or validity of these questionnaires has not been reported. In older adults with PD, one study modified a healthy older adult falls questionnaire to capture information on falls [23], but the test-retest reliability of this questionnaire was not performed. Caution is warranted when using questionnaires which are non-PD specific as they may omit key disease-related information such as the use of specific medications, date since diagnosis and/or information related to freezing episodes. Furthermore, many of these questionnaires do not assess or provide an adequate measure of near-falls [17, 32-34], which is important since a history of near-falls is a predictor of future falls in older adults with PD [34]. Therefore, there is a need for a PD-specific questionnaire that can reliably capture falls and near-falls data so that future falls prevention programs can be tailored more precisely towards the needs of older adults with PD.

Although most falls-specific questionnaires have traditionally been administered using the pen and paper method, this method has some limitations including the cost to administer and enter the data from such questionnaires, the time taken for participants to respond and potential limitations related to population reach [35].
As a result, online questionnaires may represent a viable alternative as they can overcome many of these limitations [35, 36]. Two meta-analyses have indicated that both online and paper versions of the same questionnaire yield largely comparable results for self-reported healthrelated outcomes across both general (e.g. Short Form 36 Health Survey [SF-36]) and disease-specific instruments (e.g., health and quality of life status Parkinson's Disease Questionnaire [PDQ-39]) [37, 38]. However, some studies have shown subtle differences in the responses between modes, with online questionnaires favouring slightly better health-related outcomes [39-41]. Therefore, this study aimed to develop a PD-specific falls questionnaire, using a step-wise design approach [42, 43], and to test its reliability in two modes (online and paper) among older adults with PD. Test-retest analysis was used to determine the reliability of questions concerning the number and circumstances (e.g., preceding activities, location and direction) underpinning falls and near-falls events, as well as the consequences (e.g., injuries and medical treatment) of falls experienced by older adults with PD over the previous 12-months.

\section{Methods \\ Questionnaire design}

Approval for this study was granted by the Deakin University Human Research Ethics Committee (HEAG-H 32_2016). The PD falls questionnaire (PDF-Q) was constructed using a step-wise strategy previously used for the development of reliable questionnaires $[42,43]$. This process comprised three phases: (1) development of the questionnaire domains; (2) development of the questions within each domain; and (3) test-retest reliability in a cohort of older adults with PD.

\section{Phase 1: development of the questionnaire domains}

Questionnaire domains were developed based on previous studies exploring falls circumstances among geriatric populations [12, 27, 44], and were subsequently discussed by an expert team of researchers with experience in PD and falls research. This process has been recommended previously to establish content validity of an instrument $[42,43,45]$. A consensus was reached via group discussion regarding the four primary domains of the questionnaire, which were identified as most important when understanding falls experienced by older adults with PD: 1) falls and near-falls history; 2) circumstances of falls; 3) circumstances of near-falls, and 4) consequences of falls. A PD-relevant domain was also designed to include questions on medication, date since diagnosis and freezing episodes. Finally, descriptive questions on general anthropometric (e.g., height and weight) and personal information (e.g., date of birth) were added as general 
information domain. Both the PD-relevant and the general information domains were added to provide the opportunity for further analyses on potential correlates of falls/near-falls experienced by older adults with PD.

\section{Phase 2: development of the questions within each domain}

From the studies exploring falls circumstances among geriatric populations [12, 27, 44], as well as one study for older adults with PD [22], an initial repository of falls questions was compiled that were most pertinent to capturing falls information from older adults with PD. These studies either listed specific questions within their methodology or referenced a particular questionnaire that was used to capture fall information (e.g., the New South Wales Falls Prevention Baseline Survey [27]). The questions were then narrowed down if they addressed the prevalence or incidence, circumstances and consequences of falls, and were appropriately modified for inclusion in the PDF-Q under each of the relevant domains. In addition, to maintain internal consistency of the instrument, the 'circumstance of falls and near-falls' questions were re-adapted based on the recommendations from Stack and Ashburn [24]. All falls-related questions were delineated by location (e.g., indoor or outdoor falls) to capture specific information on how and where falls occurred. Questions to capture information about near-falls were also added, with a clear definition provided to define a near-fall (e.g., "any incident where you may have fallen but were able to catch yourself and did not come to rest on the ground") [34]. As older adults with PD fall multiple times per year [5, 9, 24], we decided to capture details on the circumstances surrounding the four most recent falls and near-falls events only to avoid potential problems with recall [46]. Once a bank of questions was established, each question within each domain was reviewed, revised, and finalised via consensus by the same expert team of researchers for inclusion within the PDF-Q. Before conducting test-retest analysis, the PDF-Q was pilot tested by three individuals (all male) with $\mathrm{PD}$ (mean $\pm \mathrm{SD}$, age $70.2 \pm 4.6$ years; mild-tomoderate PD [Hoehn and Yahr score 2-3]), who were previously enrolled in a clinical exercise trial at Deakin University [47], and who all had experienced a fall(s) in the previous 12 -months. These individuals were asked to complete the questionnaire and provide feedback on the domains and content of the questions. Specifically, we asked for feedback on their general understanding of what was being asked for each question, the flow and layout of the questionnaire, and if any difficulties were found in understanding or answering the questions. This end-user feedback was used to modify and refine the PDF-Q before conducting the test-retest evaluation using both a paper and online version of the questionnaire. The final version of the PDF-Q contained six domains: the first domain consisted of general information (e.g., age, consent and person completing the questionnaire) and anthropometric questions, the second domain consisted of disease status questions, and the remaining four domains consisted of falls and near-falls questions (Additional file 1).

\section{Phase 3: establishing test-retest reliability}

Participants were included in this study if they were medically diagnosed with having PD, taking PD medication, and not receiving deep brain stimulation. Participants were excluded if they had not experienced a fall or nearfall in the previous 12-months. Furthermore, participants were asked to complete the Montreal Cognitive Assessment and a cut-off score $<26$ of 30 was used to indicate the presence of mild cognitive impairment, which precluded participation in this study.

This study implemented a two-part recruitment process for the online and paper-based questionnaires, which began after pilot testing. To assess test-retest reliability of either the online or paper-based questionnaires, participants were asked to complete the PDF-Q twice, four-weeks apart. A four-week washout period is recommended as an acceptable time period to avoid memory response bias [48]. The time to complete either questionnaire was $\sim 20 \mathrm{~min}$. For the online questionnaire, participants were initially recruited from a local community support group where a member of the research team $(\mathrm{DMH})$ conducted a short presentation about the project. From this community support group, $44 \mathrm{mem}$ bers expressed an interest in participating in the study and their contact details were recorded. After a follow up phone call, 30 members met the inclusion criteria and consented to participate and were subsequently emailed an e-link directing them to the online PDF-Q questionnaire to complete via SurveyMonkey ${ }^{\mathrm{TM}}$ (Palo Alto, CA 94301, USA, http://www.SurveyMonkey.com), with instructions on when to complete the test and retest questionnaire. For the paper-based questionnaire, 36 older adults with PD were contacted who had previously participated in a research project [35] conducted by our team and had expressed an interest in participating in future studies. From these participants, 16 met the inclusion criteria and consented to participate, and were subsequently asked to complete the paper-based PDF-Q (test and re-test) on-site at Deakin University. Overall, all 46 participants completing the online $(n=30)$ or paperbased $(n=16)$ questionnaire responded to both the test and retest questionnaire. While most responses were selfcompleted by the participants (online and paper $n=41$ [89\%]; online $n=25$ [83\%], paper $n=16$ [100\%]), some 
participants completing the online questionnaire submitted responses via proxy (e.g., personal carer) owing to an inability to complete the questionnaire themselves due to typing difficulties $(n=5[11 \%])$. In this regard, the proxy respondents were instructed to list the falls responses based on the information provided directly by the participant only and were required to do the same at retest.

\section{Statistical analysis}

Statistical analysis was conducted using Stata/SE 15 (STATA, College Station, TX, USA). Independent t-test for continuous variables and chi-square tests for categorical variables were used to compare demographic, physical and disease status characteristics between participants that completed the paper and online version of the questionnaire. Test-retest reliability for the PDF-Q was assessed by comparing the agreement between the first and second administered questionnaires for the online and paper-based questionnaire separately and both combined. Levels of agreement were determined using the Kappa ( $\mathrm{k}$ ) statistic (with standard error) for each fall and near-fall individual questions within the PDF-Q. For ordinal response variables, weighted $\kappa$ was calculated using the default weighting matrix provided in Stata/SE 15. To provide an estimate of the reliability of each domain, the individual $\mathrm{k}$ scores from each question within each domain were averaged to provide an overall $\kappa$ score of the domain. The first 10 questions of the PDF-Q consisted of standard physical characteristic and disease-specific items, which did not relate specifically to falls or near-falls and were therefore excluded from the test-retest examination to eliminate a false test-retest reliability outcome. All participants were instructed to respond to all questions. However, for the test-retest analysis, any responses of "do not remember", or unanswered questions, were subsequently coded as missing. The following standards were used to interpret the level of agreement for the $\mathrm{\kappa}$ coefficient between items: $\quad 0-0.20=$ None, $\quad 0.21-0.39=$ Minimal, $\quad 0.40-$ $0.59=$ Weak, $0.60-0.79=$ Moderate, $0.80-0.90=$ Strong, $>0.90=$ Almost Perfect [49].

\section{Sample size}

Previous research suggests that sample sizes of at least 25 participants for test-retest reliability experiments are needed to meet the confidence interval bound ratio requirements [46]. It has also been recommended that sample sizes of $\geq 30$ are needed to demonstrate adequate precision of the estimate of agreement of $\kappa$ [49]. Due to the small number of participants that we were able to recruit to complete the paper-based questionnaire, and to demonstrate adequate precision of the estimate of agreement of $\kappa$, the focus of the results will be on the test-retest reliability outcomes of the pooled $(n=46)$ responses (combined online and paper-based questionnaire data). However, for transparency and descriptive purposes, we have reported the findings from each questionnaire separately.

\section{Results}

Characteristics of participants completing the paper and online versions of the questionnaire and all participants combined are reported in Table 1 . On average, participants were aged 71 years and were a mean 9.3 years since

Table 1 Demographic and physical characteristics, and disease status of older adults with PD

\begin{tabular}{|c|c|c|c|}
\hline & Online $(n=30)$ & Paper $(n=16)$ & Combined $(n=46)$ \\
\hline \multicolumn{4}{|l|}{ Questionnaire Details } \\
\hline \multicolumn{4}{|l|}{ The person who completed the survey } \\
\hline Self-completed, n (\%) & $25(83 \%)$ & $16(100 \%)$ & $41(89 \%)$ \\
\hline Carer, n (\%) & $5(17 \%)$ & $0(0 \%)$ & $5(11 \%)$ \\
\hline \multicolumn{4}{|l|}{ Physical Characteristics } \\
\hline Age (years) & $68.6 \pm 6.7$ & $73.3 \pm 3.7$ & $71.2 \pm 6.1$ \\
\hline Height (cm) & $166.2 \pm 3.6$ & $169.1 \pm 2.6$ & $168.1 \pm 2.9$ \\
\hline Weight (kg) & $67.8 \pm 5.6$ & $65.5 \pm 4.9$ & $67.1 \pm 4.8$ \\
\hline Body mass index $\left(\mathrm{kg} / \mathrm{m}^{2}\right)$ & $24.6 \pm 3.4$ & $22.9 \pm 4.1$ & $23.7 \pm 3.5$ \\
\hline Sex, n (\% male $)^{\mathrm{a}}$ & $20(67 \%)$ & $10(63 \%)$ & $30(65 \%)$ \\
\hline \multicolumn{4}{|l|}{ Parkinson's disease status } \\
\hline Years since formal diagnosis & $10.2 \pm 2.6$ & $8.3 \pm 2.2$ & $9.3(2.4)$ \\
\hline Taking medication for PD, n (\% yes) & $30(100 \%)$ & $16(100 \%)$ & $46(100 \%)$ \\
\hline
\end{tabular}

All data are means \pm standard deviation (SD) unless otherwise stated (i.e. number [percentage])

PD Parkinson's disease

${ }^{\text {a }}$ Sixteen participants did not disclose information on gender 
formal PD diagnosis. No statistical differences were observed between participants completing the paper and online version of the questionnaire.

Descriptive statistics and test-retest agreement for each question of the PDF-Q are provided for falls (Table 2), near-falls (Table 3 ) and injurious falls (Table 4) with the findings for online, paper and combined versions of the questionnaires presented. As indicated previously, due to the small number of participants that completed the paper-based questionnaire $(n=16)$, the focus of the results is on the test-retest reliability outcomes of the pooled responses from both questionnaires combined $(n=46)$. However, for the majority of the individual questions, there was comparable test-retest reliability for the online and paper-based questionnaire. Furthermore, only data for the participants' two most recent falls and nearfalls were included as most participants either could not remember the details of the events beyond the two most recent falls and near-falls $(n=29,63 \%)$ or did not have more than two falls or near-falls $(n=12,26 \%)$.

The overall average $\kappa$ score for the fall's circumstances (location, activity, how, and direction) domain based on the pooled questionnaire results was 0.79 , which represented moderate agreement. As shown in Table 2, the test-retest agreement was generally moderate to strong for each question related to falls. The question concerning the number of falls in the past 12 months demonstrated weak agreement $(\kappa=0.41)$, but the questions concerning the location of falls $(\kappa=0.89)$ and the number of indoor $(\kappa=0.86)$ and outdoor $(\kappa=0.75)$ falls all demonstrated moderate to strong agreement. For the most recent indoor and outdoor fall, there was moderate to strong agreement for the questions related to the location (indoor fall $1 \kappa=0.74$; outdoor fall $1 \kappa=0.76$ ), direction (indoor fall $1 \kappa=0.80$; outdoor fall $1 \kappa=0.81$ ), activity (indoor fall $1 \kappa=0.70$; outdoor fall $1 \kappa=0.82$;), and cause (indoor fall $1 \kappa=0.75$; outdoor fall $1 \kappa=0.62$;). Similar results were observed for the second most recent indoor and outdoor fall questions regarding the circumstances (location, activity, how and direction) (Table 2).

All participants reported having a near-fall in the previous 12 -months, and therefore $\mathrm{k}$ values were not presented for the first question. The overall $\mathrm{k}$ score for the near-fall circumstances (location, activity, how, and direction) domain for the pooled questionnaire results was 0.60 , which represented moderate agreement. Questions assessing the number of near-falls in the previous 12 -months demonstrated weak agreement $(\kappa=0.51)$, while questions concerning the location of near-falls demonstrated almost perfect agreement $(\kappa=1.0)$ (Table 3). In contrast, there was a weak agreement for questions related to the number of indoor $(\kappa=0.47)$ and outdoor $(\kappa=0.56)$ near-falls, while the direction (near-fall $1 \kappa=0.54$ ), activity (near-fall $1 \kappa=0.65$ ) cause (near-fall $1 \kappa=0.56$ ) of near-falls questions all demonstrated between weak to moderate agreement (Table 3). Once more, similar results were observed for the circumstances (location, activity, how and direction) of the second most recent near-falls questions (Table 3).

The overall average $\mathrm{k}$ score for the injurious falls domain based on the pooled questionnaire results was 0.73 , which represented moderate agreement. As shown in Table 4, the test-retest agreement was moderate to strong for the individual questions concerning whether participants experienced an injurious fall in the past 12 -months $(\kappa=0.90)$, the number of injurious falls (indoor $\kappa=0.59$ ), number of visits to a healthcare professional (indoor $\kappa=0.65$ ), injuries sustained as a result of an injurious fall (fall $1 \kappa=0.57$; fall $2 \kappa=0.75$ ) and medical treatment sought for any injurious fall $(\kappa=0.89)$. Due to only one person in this cohort experiencing a fracture, the $\mathrm{k}$ values were not provided for fracture-related questions. Similarly, only one person reported experiencing an injurious outdoor fall, and so the $\mathrm{k}$ values for these questions were also not provided.

\section{Discussion}

To our knowledge, the PDF-Q used in this study is the first falls-specific questionnaire to be developed and tested for reliability among older adults with PD. The main finding from this study was that the pooled results from the online and paper modes for the PDF-Q collectively demonstrated moderate to strong agreement (reliability) scores for most questions related to the circumstances and consequences of falls and near-falls, though there was a weaker agreement for the number of falls and near-falls questions. These findings indicate that the PDF-Q represents a reliable tool to capture the circumstances and consequences of falls and near-falls in older adults with PD, yet the recall length (previous 12-months) may be too long to accurately remember the number of falls and near-falls sustained.

Since there is no previous data on the reliability of PDspecific falls-related questionnaires, it is difficult to make direct comparisons with our test-retest results. In nonPD older adults, there is data on the test-retest reliabilities of both the Carefall Triage Instrument [50] and the Falls Efficacy Scale-International (FES-I) [51]. The Carefall Triage Instrument demonstrated minimal to strong test-retest reliability for self-rated questions assessing falls risk among 27 older adults aged over 65 years, while the FES-I has demonstrated strong test-retest reliability for questions related to concerns about falling among 16 older adults aged between 60 and 95 years. However, due to the diverse statistical approaches implemented (e.g., the FES-I was analysed using Cronbach's $\alpha$ and intraclass 


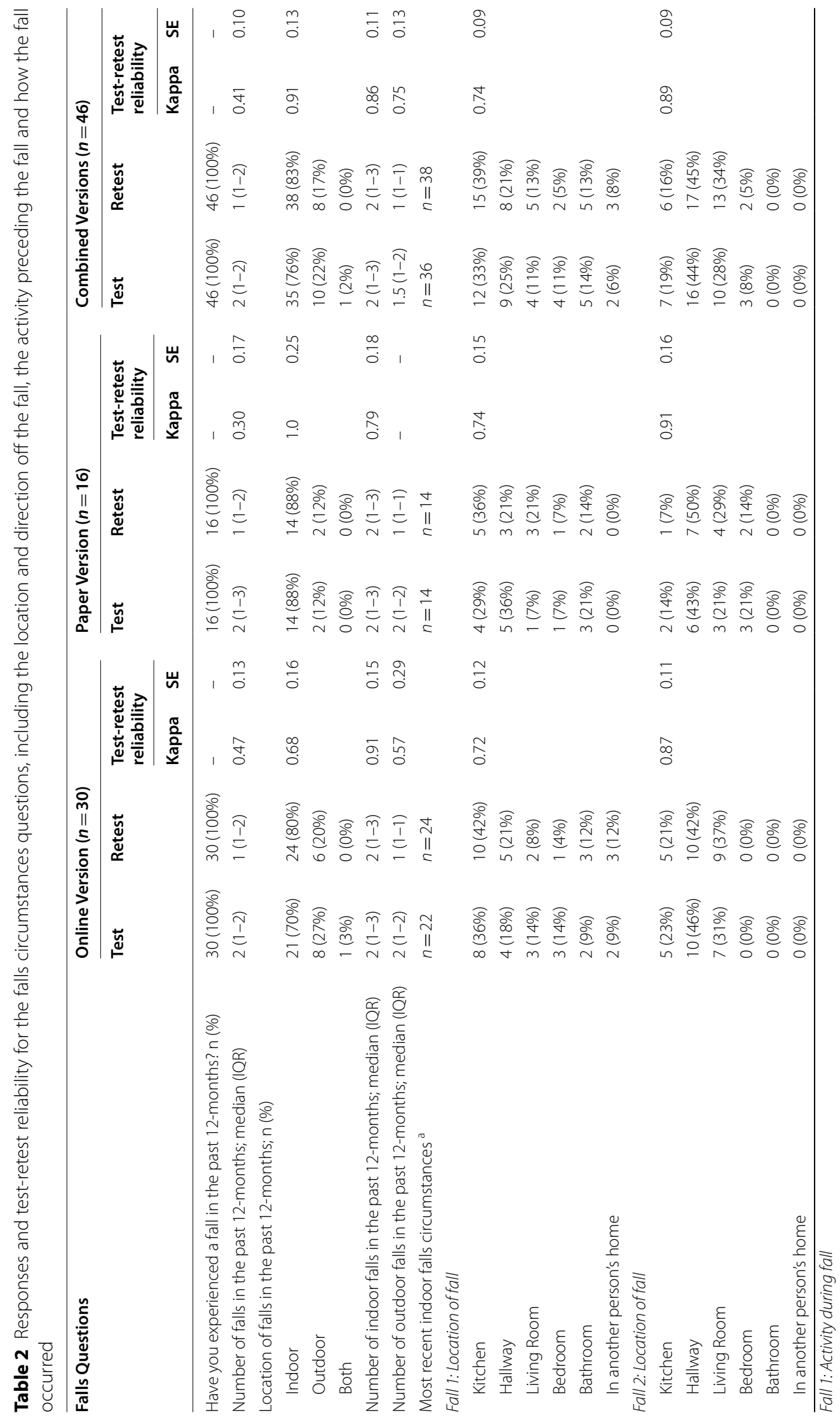




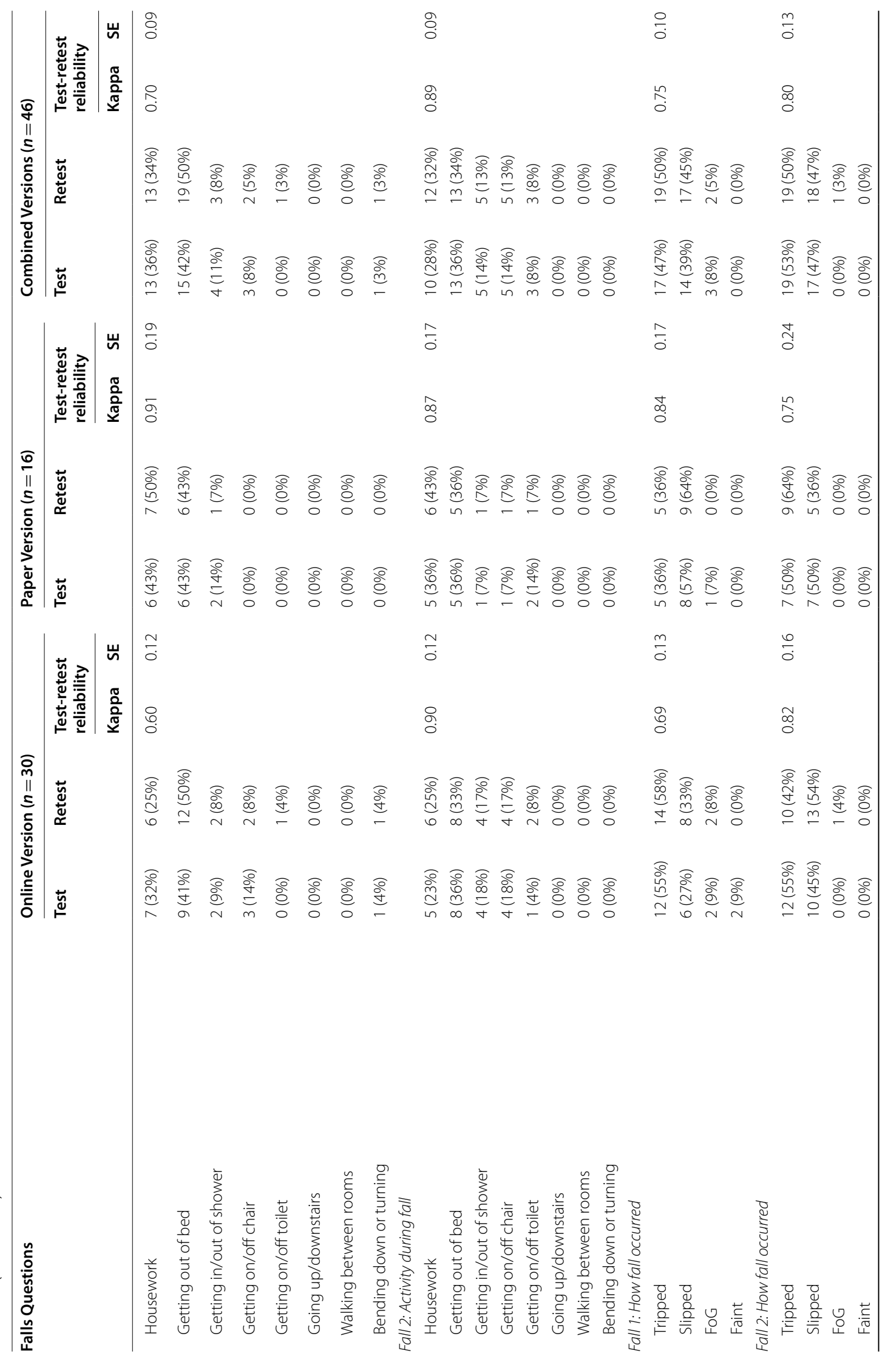




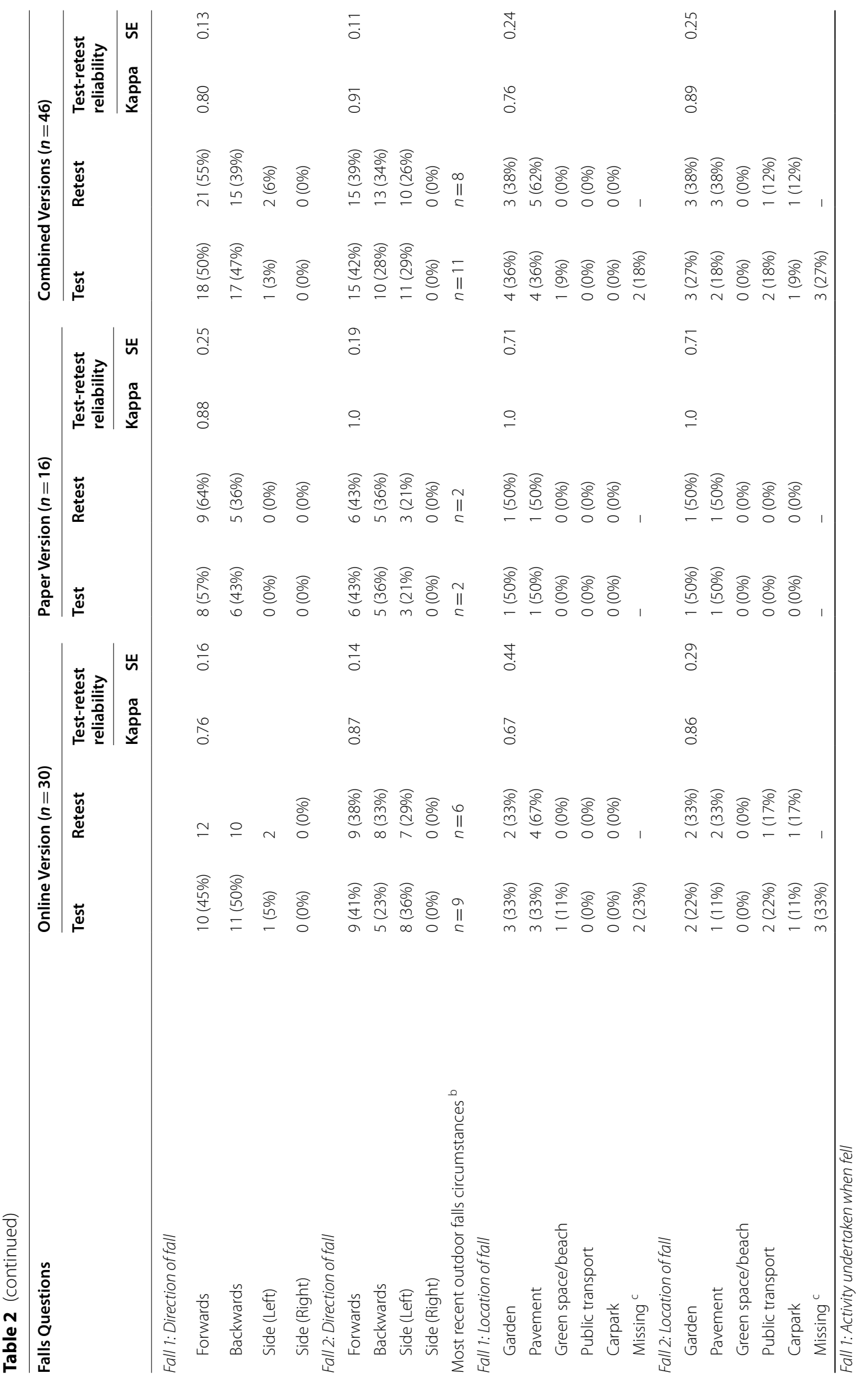




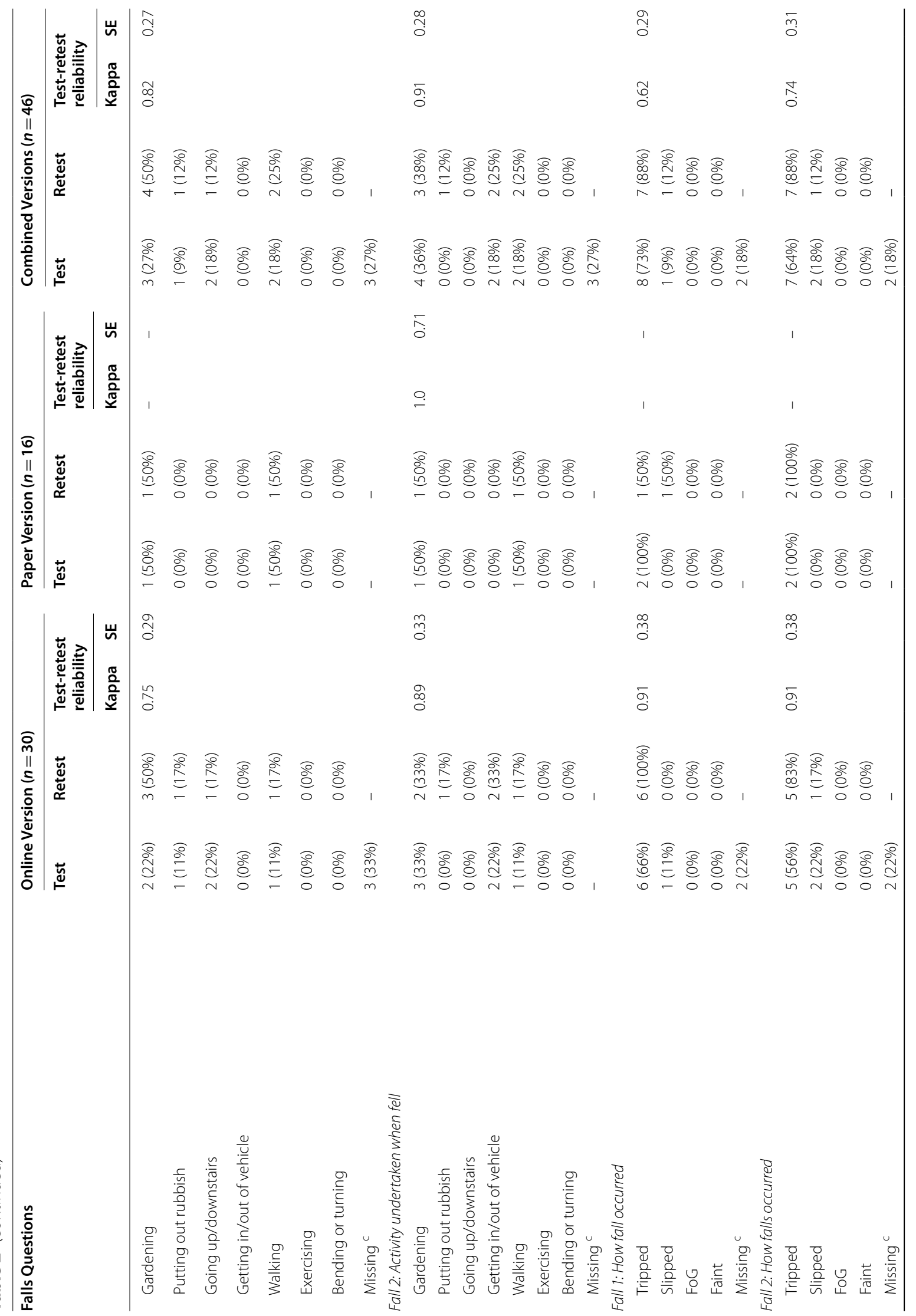




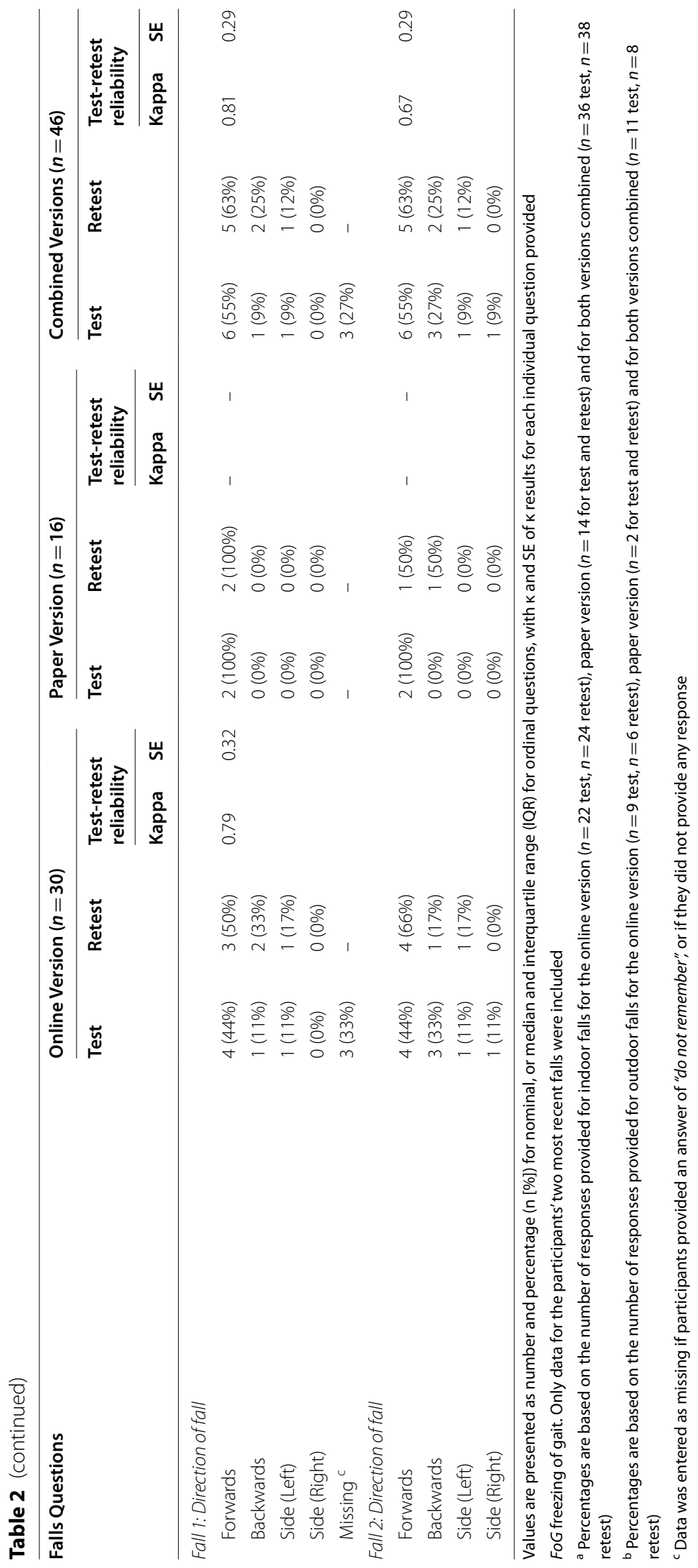




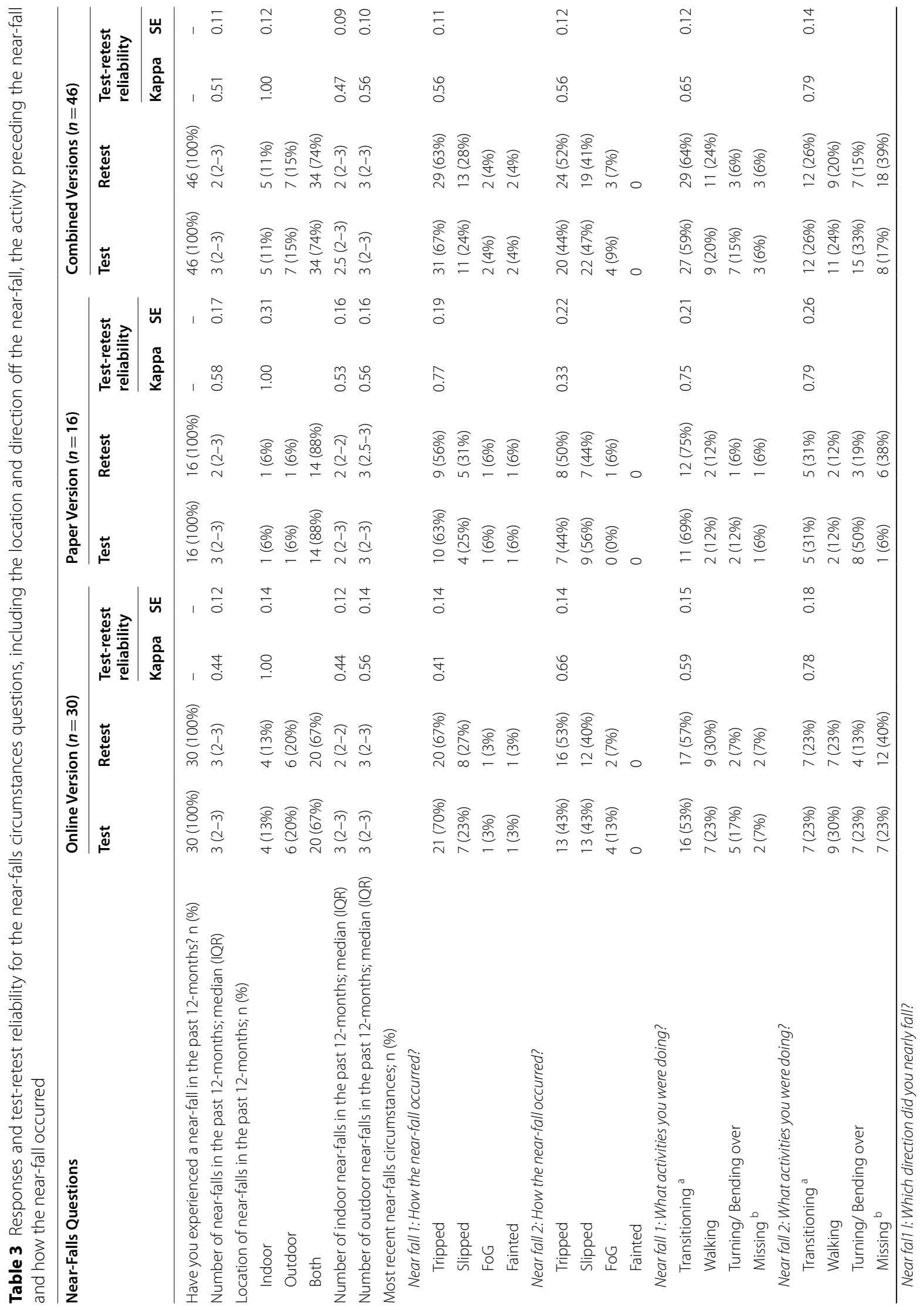




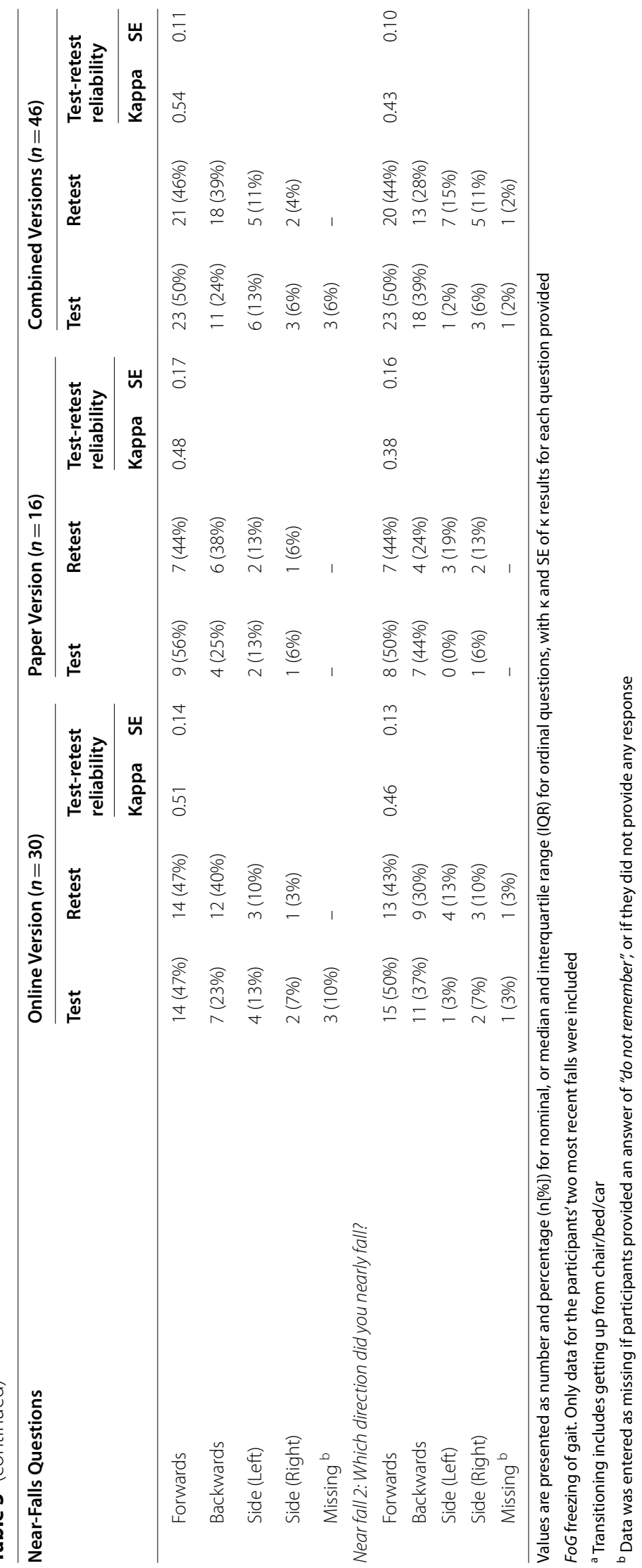




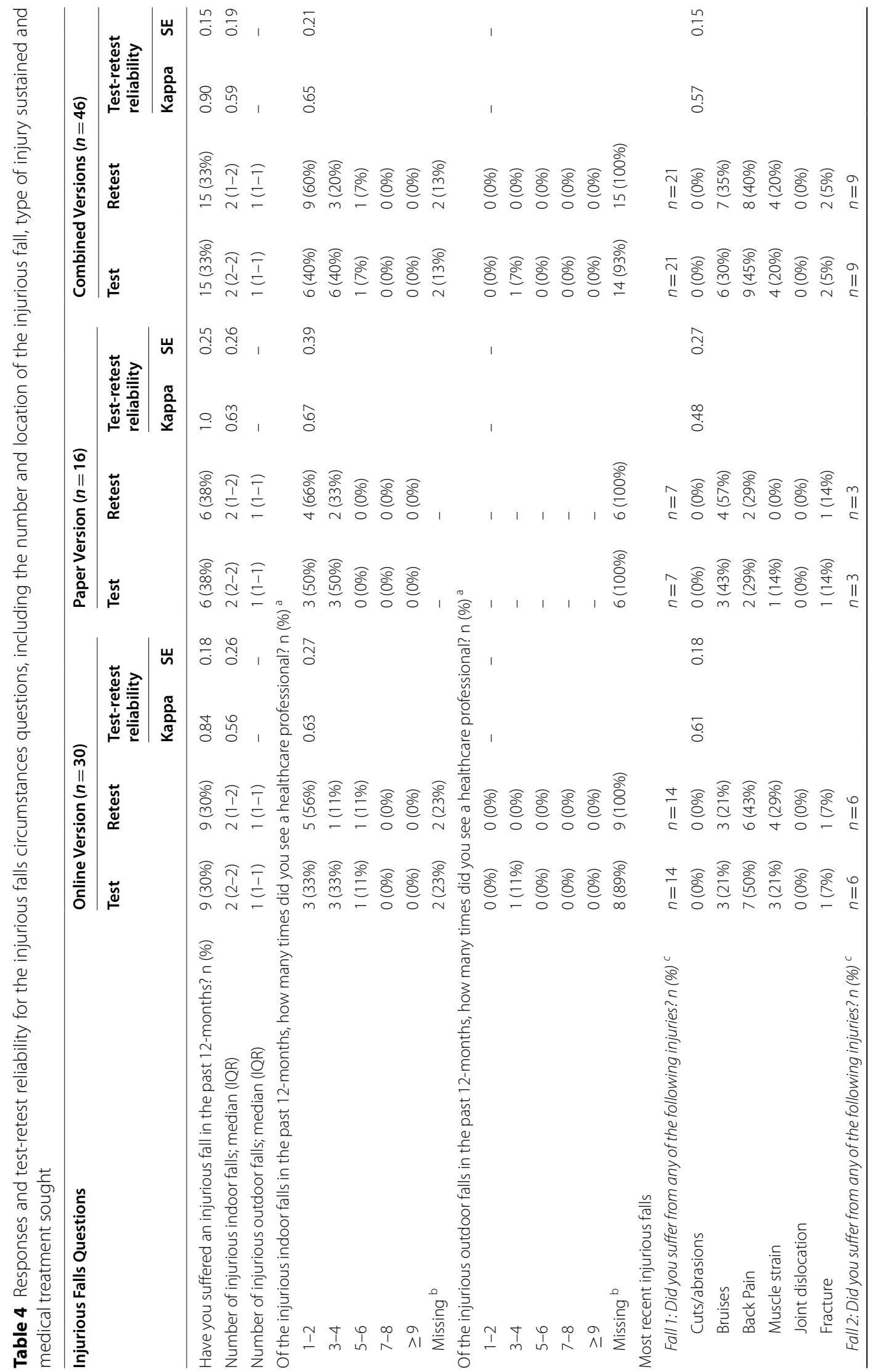




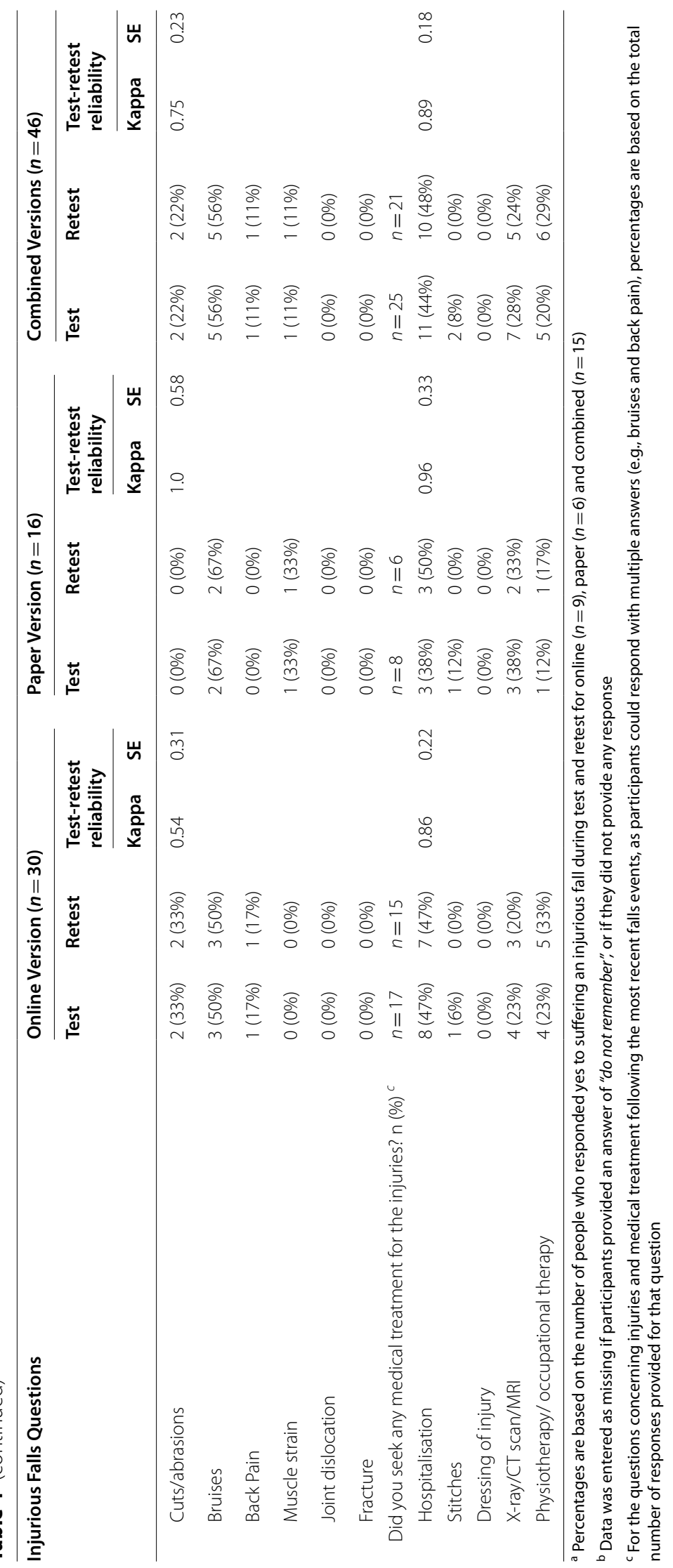


correlation coefficients) and the differences between domain designs of the The Carefall Triage instrument and the FES-I compared to the PDF-Q, it is difficult to directly compare the reliability results of each questionnaire concerning outcomes such as the number, circumstances and consequences of falls. Furthermore, the PDF-Q developed in this study is the first of its kind to establish the test-retest reliability of questions capturing near-falls information among older adults with PD. To our knowledge, no studies have evaluated the test-retest reliability of a near-falls questionnaire, and our pooled $\mathrm{k}$ results for near-falls indicates that there was generally a moderate level of agreement for these questions.

As a comparison between the pooled PDF-Q domains for falls and near-falls, there are some potential explanations for why the near-falls circumstances domain $(\kappa=0.60)$ demonstrated relatively weaker reliability than the circumstances of the falls domain $(\kappa=0.79)$, although both domains still demonstrated moderate reliability. Firstly, near-fall events may be a less traumatic experience compared to an actual fall, particularly if the individual experiences an injury as a result of the fall $[52,53]$. Indeed, we established a strong agreement for the question concerning whether participants received medical treatment for injury following a fall $(n=21$, $\kappa=0.89$ ). Thus, it may be that older adults with PD are more likely to recall the circumstances of events preceding a fall resulting in injury, compared to near-falls where injuries are less likely. Jayasinghe et al., [54] showed that $27 \%$ of older adult (non-PD) fallers (from a sample of 100) had post-traumatic stress as a result of the fall, and $78 \%$ reported 'feeling emotionally upset when reminded of the fall'. It is also possible the 12-month recall period was too long for the participants to recall the circumstances of near-falls, especially given they are typically not traumatic events. Certainly, there is evidence that older adults may have difficulty recalling non-traumatic near-fall events beyond 3-months $[53,55]$, which may be worse in older adults with PD whom often have deficits in working memory, or mild cognitive impairments [53, 56]. For instance, it has been reported that $45 \%$ of older adults with PD had memory deficits compared to healthy age-matched controls [57]. However, cognitive deficits are unlikely to explain the disproportionate near-falls and falls reliability results in this study as participants with MCI were excluded. Finally, it may have been difficult for the older adults in this study to differentiate near-falls events from stumbles/trips, where a fall was not going to occur even without the support of a wall or railing etc. [17]. In the PDF-Q we adopted the definition of a nearfall from Maidan et al., [58] and Lindholm et al., [34] which was defined as "any incident where you may have fallen but were able to catch yourself and did not come to rest on the ground". Although this definition may be open to interpretation, we were still able to demonstrate mostly moderate levels of agreement for each question within the near-falls domain. As such, despite the relatively weaker reliability scores compared to the circumstances of the falls domain, we believe that the PDF-Q represents a reliable tool to capture near-falls information in older adults with PD.

The reliability results for the questions concerning the number of falls and near-falls showed relatively weaker agreement compared to the circumstances and location of falls and near-falls questions. Previous studies have indicated that recall length using retrospective surveys should focus on shorter periods (e.g., less than 3-months) to reduce memory bias and improve the precision of the responses $[46,50]$. As such, this may explain the poorer reliability scores for these questions which focussed on recalling the number of falls and near-falls events over the previous 12-months. However, further research is needed investigating the reliability of these questions over shorter recall lengths to confirm this contention.

The pooled results for the injurious falls domain demonstrated moderate to strong agreement for each question within the PDF-Q. To our knowledge, no studies have reported test-retest reliability for injurious falls among older adults with PD. In this study, data from 15 participants were used for the test-retest analysis on the number and circumstances of injurious falls in the past 12-months. As such, the reliability results for the injurious falls questions must be interpreted with caution, as it has been reported that at least 25 participants are required for acceptable test-retest reliability analysis [38]. Nevertheless, the reliability results indicating a strong agreement may be generalisable for larger cohorts of older adults with PD who suffer injurious falls. For example, our participants, on average, had similar characteristics, and reported a comparable injurious falls rate (33\%), to previous epidemiology studies investigating injurious falls among older adults with PD [19, 22, 59]. Still, further research should consolidate our reliability results among a larger sample $(n \geq 25)$ of individuals with PD who have suffered an injurious fall(s) to strengthen these findings.

A strength of this study was the step-wise development of the PDF-Q, which included a literature search and expert consensus regarding the questions and domains of the instrument. However, there are several limitations associated with this study that must be acknowledged. Firstly, we experienced difficulties in recruiting older adults with PD to complete the paper-based version of the PDF-Q, and due to this small sample size $(n=16)$ we were unable to directly compare the test-retest reliability findings with the online version. Nevertheless, it is worth noting that for many of the individual questions there 
was a similar level of agreement (reliability) between the online and paper-based version. Consistent with this observation, several previous studies have shown comparable responses (and similar reliabilities) between paper and online questionnaires among healthy populations [40, 60-62]. Additionally, one study compared online and paper versions of the 39-item self-report Parkinson's disease questionnaire (PDQ-39) among 44 older adults with PD, and found little differences between modes regarding responsiveness and data precision [63]. Second, a four-week wash-out period between testing and retesting days was adopted in this study. Although this is standard practice for test-retest reliability analysis, we did not capture any information about falls or near-falls events within these 4 weeks (e.g., via falls diaries). Given that older adults with PD are a high-risk group for recurrent falls $[9,64]$, it cannot be determined if the reported circumstances surrounding falls and near-falls between the test and retest periods are accurately assessing the same 'most recent' fall/near-fall event following the fourweek washout period. However, we postulate that given the acceptable levels of agreement scores for the majority of questions, many of the most recent falls/nearfalls remained the same even after 4 weeks. Third, we acknowledge that with the falls and near-falls domains some descriptive questions presented lower agreement scores compared to others, which may have been the result of inadequate instruction; particularly concerning the order of the most recent events. However, given the high number of "don't remember" responses from participants for falls/near-falls 3 and 4 compared to falls/nearfalls 1 and 2, we suspect that participants interpreted fall/ near-fall 1 as being their most recent event and fall/nearfall 2 as their second most recent, and so on. Indeed, it may be that older adults with PD find it difficult to recall falls/near-falls events beyond the two most recent events $[52,53]$. Researchers and clinicians using the PDF-Q should, therefore, consider reducing the recall period for more reliable responses.

\section{Conclusions}

In conclusion, the new PDF-Q developed in this study was found to be reliable for capturing the circumstances and consequences of recent falls and near-falls in older adults with PD, but showed weaker agreement for the questions concerning the number of falls and near-falls in the previous 12 -months. It is possible that the recall period was too long for participants to accurately remember the number of falls and near-falls events experienced, though more research is needed to investigate the reliability of these questions using shorter recall lengths to confirm this. There was also a comparatively weaker agreement between the near-falls circumstances domain and the circumstances of the falls domain of this questionnaire. This is possibly due to older adults with PD being less able to precisely recall the events surrounding near-falls, which are generally less traumatic compared to falls. However, further exploration is needed comparing the precision of recalling the events surrounding near-falls to falls among older adults with PD to verify our contention. The step-wise design of the PDF-Q was sufficient in establishing content validity of the instrument. Despite this, future studies should aim to establish criterion and construct validities of the PDF-Q. Overall, this is the first attempt of any kind to develop and demonstrate test-retest reliability of a PD-specific falls questionnaire, which will serve as a valuable tool for accurately capturing falls, near-falls and injurious falls information from older adults with PD.

\section{Abbreviations}

PD: Parkinson's disease; PDF-Q: Parkinson's diease falls questionnaire; PDQ-39: 39-item self-report Parkinson's disease questionnaire; FRQ: Falls Risk Questionnaire; FES-I: Falls Efficacy Scale-International; SF-36: Short Form 36 Health Survey.

\section{Supplementary Information}

The online version contains supplementary material available at https://doi. org/10.1186/s12877-021-02555-6.

\section{Additional file 1.}

\section{Acknowledgements}

We would like to acknowledge, and thank, those participants who gave up their time to be part of this study.

\section{Authors' contributions}

DMH, RLD, WPT, LJ and TR all helped to construct the PDF-Q. DMH recruited the participants, tested the PDF-Q, analysed and interpreted the participant data. DMH drafted the initial manuscript and tables, and RMD, GA, WPT, LJ and RLD and TR were major contributors in reviewing the manuscript. All authors read and approved the final manuscript.

\section{Funding \\ During the conducting of this study, DMH was supported by the Deakin University Post Graduate Research Scholarship (DUPGR) and WPT was sup- ported by an Alfred Deakin Postdoctoral Research Fellowship. The funding did not have any role in the design and execution of the study, the collection, analysis or interpretation of data, the decision to submit results nor in writing the manuscript.}

\section{Availability of data and materials}

The datasets analysed during the current study are available from the corresponding author on reasonable request.

\section{Declarations}

Ethics approval and consent to participate

Approval for this study was granted by the Deakin University Human Research Ethics Committee (HEAG-H 32_2016). After reading a plain language statement, written informed consent was provided by all participants before undertaking the PDF-Q. 


\section{Consent for publication}

Not applicable.

\section{Competing interests}

The authors declare that they have no competing interests.

\begin{abstract}
Author details
${ }^{1}$ Institute for Physical Activity and Nutrition (IPAN), School of Exercise and Nutrition Sciences, Deakin University, Geelong, Australia. ${ }^{2}$ First Year College, Victoria University, Melbourne, Australia. ${ }^{3}$ Department of Medicine-Western Health, University of Melbourne, St Albans, VIC 3021, Australia. ${ }^{4}$ Australian Institute for Musculoskeletal Science (AIMSS), University of Melbourne and Western Health, St Albans, VIC 3021, Australia. ${ }^{5}$ Physiotherapy Department, Epworth HealthCare, Melbourne, Australia. ${ }^{6}$ School of Behavioural and Health Sciences, Australian Catholic University, Melbourne, Australia. ${ }^{7}$ Faculty of Sport and Health Sciences and Gerontology Research Center, University of Jyväskylä, Jyväskylä, Finland. ${ }^{8}$ Physical Education and Sports Science Academic Group, National Institute of Education, Nanyang Technological University, Singapore, Singapore.
\end{abstract}

Received: 17 September 2020 Accepted: 14 October 2021 Published online: 30 October 2021

\section{References}

1. Burns ER, Stevens JA, Lee R. The direct costs of fatal and non-fatal falls among older adults—United States. J Saf Res. 2016;58:99-103.

2. Stel VS, Smit JH, Pluijm SM, Lips P. Consequences of falling in older men and women and risk factors for health service use and functional decline. Age Ageing. 2004;33(1):58-65.

3. Stevens JA, Corso PS, Finkelstein EA, Miller TR. The costs of fatal and nonfatal falls among older adults. Inj Prev. 2006;12(5):290-5.

4. Paul SS, Harvey L, Canning CG, Boufous S, Lord SR, Close JCT, et al. Fallrelated hospitalization in people with Parkinson's disease. Eur J Neurol. 2017;3:523.

5. Matinolli M, Korpelainen J, Sotaniemi K, Myllylä V, Korpelainen R. Recurrent falls and mortality in Parkinson's disease: a prospective two-year follow-up study. Acta Neurol Scand. 2011;123(3):193-200.

6. Fasano A, Canning CG, Hausdorff JM, Lord S, Rochester L. Falls in Parkinson's disease: a complex and evolving picture. Mov Disord. 2017:32(11):1524-36.

7. Bergen $\mathrm{G}$. Falls and fall injuries among adults aged $\geq 65$ years-United States, 2014. MMWR Morb Mortal Wkly Rep. 2016;65(37):993-8.

8. Cigolle CT, Ha J, Min LC, Lee PG, Gure TR, Alexander NB, et al. The epidemiologic data on falls, 1998-2010: more older Americans report falling. JAMA Intern Med. 2015;175(3):443-5.

9. Allen NE, Schwarzel AK, Canning CG. Recurrent falls in parkinson's disease: a systematic review. Parkinsons Dis. 2013;2013:1-16.

10. Terroso M, Rosa N, Torres Marques A, Simoes R. Physical consequences of falls in the elderly: a literature review from 1995 to 2010. Eur Rev Aging Phys Act. 2014;11(1):51-9.

11. Rubenstein LZ. Falls in older people: epidemiology, risk factors and strategies for prevention. Age Ageing. 2006;35(suppl_2):ii37-41.

12. Stevens JA, Mahoney JE, Ehrenreich $\mathrm{H}$. Circumstances and outcomes of falls among high risk community-dwelling older adults. Inj Epidemiol. 2014;1(1):5.

13. Magrinelli F, Picelli A, Tocco P, Federico A, Roncari L, Smania N, et al. Pathophysiology of Motor Dysfunction in Parkinson's Disease as the Rationale for Drug Treatment and Rehabilitation. Parkinsons Dis. 2016;2016:9832839.

14. Berardelli A, Rothwell JC, Thompson PD, Hallett M. Pathophysiology of bradykinesia in Parkinson's disease. Brain. 2001;124(11):2131-46.

15. Rinalduzzi S, Trompetto C, Marinelli L, Alibardi A, Missori P, Fattapposta F, et al. Balance dysfunction in Parkinson's disease. Biomed Res Int. 2015;2015:1-10

16. Park J-H, Kang Y-J, Horak FB. What is wrong with balance in Parkinson's disease? J Mov Disord. 2015;8(3):109.

17. Gazibara T, Tepavcevic DK, Svetel M, Tomic A, Stankovic I, Kostic VS, et al. Near-falls in people with Parkinson's disease: circumstances, contributing factors and association with falling. Clin Neurol Neurosurg. 2017;161:51-5.

18. Gazibara T, Pekmezovic T, Kisic-Tepavcevic D, Svetel M, Tomic A, Stankovic I, et al. Incidence and prediction of falls in Parkinson's disease: a prospective cohort study. Eur J Epidemiol. 2015;30(4):349-52.

19. Gazibara T, Pekmezovic T, Tepavcevic DK, Tomic A, Stankovic I, Kostic VS, et al. Circumstances of falls and fall-related injuries among patients with Parkinson's disease in an outpatient setting. Geriatr Nurs. 2014:35(5):364-9.

20. Pelicioni PH, Menant JC, Latt MD, Lord SR. Falls in Parkinson's disease subtypes: risk factors, locations and circumstances. Int J Environ Res Public Health. 2019;16(12):2216.

21. Lamont RM, Morris ME, Menz HB, McGinley JL, Brauer SG. Falls in people with Parkinson's disease: a prospective comparison of community and home-based falls. Gait Posture. 2017;55:62-7.

22. Ashburn A, Stack E, Ballinger C, Fazakarley L, Fitton C. The circumstances of falls among people with Parkinson's disease and the use of falls diaries to facilitate reporting. Disabil Rehabil. 2008;30(16):1205-12.

23. Bloem BR, Grimbergen YA, Cramer M, Willemsen M, Zwinderman $\mathrm{AH}$. Prospective assessment of falls in Parkinson's disease. J Neurol. 2001;248(11):950-8.

24. Stack E, Ashburn A. Fall events described by people with Parkinson's disease: implications for clinical interviewing and the research agenda. Physiother Res Int. 1999;4(3):190-200.

25. Hunter $H$, Rochester L, Morris R, Lord S. Longitudinal falls data in Parkinson's disease: feasibility of fall diaries and effect of attrition. Disabil Rehabil. 2018;40(19):2236-41.

26. Diefenbach T. Are case studies more than sophisticated storytelling?: methodological problems of qualitative empirical research mainly based on semi-structured interviews. Qual Quant. 2009;43(6):875.

27. Milat AJ, Watson WL, Monger C, Barr M, Giffin M, Reid M. Prevalence, circumstances and consequences of falls among community-dwelling older people: results of the 2009 NSW falls prevention baseline survey. N S W Public Health Bull. 2011;22(4):43-8.

28. Pitchai P, Dedhia HB, Bhandari N, Krishnan D, D'Souza NRJ, Bellara JM. Prevalence, risk factors, circumstances for falls and level of functional independence among geriatric population - a descriptive study. Indian J Public Health. 2019;63(1):21-6.

29. Crenshaw JR, Bernhardt KA, Achenbach SJ, Atkinson EJ, Khosla S, Kaufman $\mathrm{KR}$, et al. The circumstances, orientations, and impact locations of falls in community-dwelling older women. Arch Gerontol Geriatr. 2017:73:240-7.

30. Sanders K, Lim K, Stuart AL, Macleod A, Scott D, Nicholson GC, et al. Diversity in fall characteristics hampers effective prevention: the precipitants, the environment, the fall and the injury. Osteoporos Int. 2017:28(10):3005-15.

31. Obrist S, Rogan S, Hilfiker R. Development and evaluation of an online fall-risk questionnaire for nonfrail community-dwelling elderly persons: a pilot study. Curr Gerontol Geriatr Res. 2016;2016:1-16.

32. Rezvani ZN, Barr E, Gruber-Baldini A, Savitt J, Von Coelln FR, Reich S, et al. Stumbling, near-falls, freezing or postural instability: what predicts falling best in Parkinson disease? Neurology. 2018;90:P2.057.

33. Kader M, Iwarsson $\mathrm{S}$, Odin P, Nilsson MH. Fall-related activity avoidance in relation to a history of falls or near falls, fear of falling and disease severity in people with Parkinson's disease. BMC Neurol. 2016;16(1):84.

34. Lindholm B, Hagell P, Hansson O, Nilsson MH. Prediction of falls and/ or near falls in people with mild Parkinson's disease. PLoS One. 2015:10(1):e0117018.

35. Lefever S, Dal M, Matthiasdottir A. Online data collection in academic research: advantages and limitations. Br J Educ Technol. 2007;38(4):574-82.

36. Hunter L. Challenging the reported disadvantages of e-questionnaires and addressing methodological issues of online data collection. Nurse Res. 2012;20(1):11-20.

37. Gwaltney CJ, Shields AL, Shiffman S. Equivalence of electronic and paperand-pencil administration of patient-reported outcome measures: a meta-analytic review. Value Health. 2008;11(2):322-33.

38. Muehlhausen W, Doll H, Quadri N, Fordham B, O'Donohoe P, Dogar N, et al. Equivalence of electronic and paper administration of patientreported outcome measures: a systematic review and meta-analysis of 
studies conducted between 2007 and 2013. Health Qual Life Outcomes. 2015;13(1):167.

39. Braekman E, Berete F, Charafeddine R, Demarest S, Drieskens S, Gisle L, et al. Measurement agreement of the self-administered questionnaire of the Belgian health interview survey: paper-and-pencil versus web-based mode. PLoS One. 2018;13(5):e0197434.

40. Touvier M, Méjean C, Kesse-Guyot E, Pollet C, Malon A, Castetbon K, et al. Comparison between web-based and paper versions of a self-administered anthropometric questionnaire. Eur J Epidemiol. 2010;25(5):287-96.

41. Zazpe I, Santiago S, De la Fuente-Arrillaga C, Nuñez-Córdoba J, BesRastrollo M, Martínez-González MA. Paper-based versus web-based versions of self-administered questionnaires, including food-frequency questionnaires: prospective cohort study. JMIR Public Health Surveill. 2019;5(4):e11997.

42. Rattray J, Jones MC. Essential elements of questionnaire design and development. J Clin Nurs. 2007;16(2):234-43.

43. Ball LE, Leveritt MD. Development of a validated questionnaire to measure the self-perceived competence of primary health professionals in providing nutrition care to patients with chronic disease. Fam Pract. 2015;32(6):706-10.

44. Duckham RL, Procter-Gray E, Hannan MT, Leveille SG, Lipsitz LA, Li W. Sex differences in circumstances and consequences of outdoor and indoor falls in older adults in the MOBILIZE Boston cohort study. BMC Geriatr. 2013;13(1):133.

45. Polit DF. Getting serious about test-retest reliability: a critique of retest research and some recommendations. Qual Life Res. 2014;23(6):1713-20.

46. Guo H, Pohl E, Gerokostopoulos A, editors. Determining the right sample size for your test: theory and application. Reliability and Maintainability Symposium; 2013.

47. Harris DM, Rantalainen T, Muthalib M, Johnson L, Duckham RL, Smith ST, et al. Concurrent exergaming and transcranial direct current stimulation to improve balance in people with Parkinson's disease: study protocol for a randomised controlled trial. Trials. 2018;19(1):387.

48. Pittman J, Bakas T. Measurement and instrument design. J Wound Ostomy Continence Nurs. 2010;37(6):603-7.

49. McHugh ML. Interrater reliability: the kappa statistic. Biochemia medica: Biochemia medica. 2012;22(3):276-82.

50. van Hensbroek PB, van Dijk N, van Breda GF, Scheffer AC, van der Cammen TJ, Lips P, et al. The CAREFALL triage instrument identifying risk factors for recurrent falls in elderly patients. Am J Emerg Med. 2009;27(1):23-36.

51. Yardley L, Beyer N, Hauer K, Kempen G, Piot-Ziegler C, Todd C. Development and initial validation of the falls efficacy scale-international (FES-I). Age Ageing. 2005;34(6):614-9.

52. Peel N. Validating recall of falls by older people. Accid Anal Prev. 2000;32(3):371-2
53. Ganz DA, Higashi T, Rubenstein LZ. Monitoring falls in cohort studies of community-dwelling older people: effect of the recall interval. J Am Geriatr Soc. 2005:53(12):2190-4

54. Jayasinghe N, Sparks MA, Kato K, Wyka K, Wilbur K, Chiaramonte G, et al. Posttraumatic stress symptoms in older adults hospitalized for fall injury. Gen Hosp Psychiatry. 2014;36(6):669-73.

55. Cummings SR, Nevitt MC, Kidd S. Forgetting falls: the limited accuracy of recall of falls in the elderly. J Am Geriatr Soc. 1988;36(7):613-6.

56. Whittington CJ, Podd J, Stewart-Williams S. Memory deficits in Parkinson's disease. J Clin Exp Neuropsychol. 2006;28(5):738-54.

57. Muslimović D, Post B, Speelman JD, Schmand B. Cognitive profile of patients with newly diagnosed Parkinson disease. Neurology. 2005;65(8):1239-45.

58. Maidan I, Freedman T, Tzemah R, Giladi N, Mirelman A, Hausdorff J. Introducing a new definition of a near fall: intra-rater and inter-rater reliability. Gait Posture. 2014;39(1):645-7.

59. Wielinski CL, Erickson-Davis C, Wichmann R, Walde-Douglas M, Parashos SA. Falls and injuries resulting from falls among patients with Parkinson's disease and other parkinsonian syndromes. Mov Disord. 2005:20(4):410-5.

60. Sekeres MA, Maciejewski JP, List AF, Steensma DP, Artz A, Swern AS, et al. Perceptions of disease state, treatment outcomes, and prognosis among patients with myelodysplastic syndromes: results from an internet-based survey. Oncologist. 2011;16(6):904.

61. Hill EL, Cumming RG, Lewis R, Carrington S, Couteur DGL. Sleep disturbances and falls in older people. J Gerontol Ser A Biol Med Sci. 2007:62(1):62-6.

62. Vallejo MA, Mañanes G, Comeche Ml, Díaz MI. Comparison between administration via internet and paper-and-pencil administration of two clinical instruments: SCL-90-R and GHQ-28. J Behav Ther Exp Psychiatry. 2008:39(3):201-8

63. Cubo E, Trejo Gabriel-Galán JM, Seco Martínez J, Rioja Alcubilla C, Yang C, Fernández Arconada O, et al. Comparison of office-based versus home web-based clinical assessments for Parkinson's disease. Mov Disord. 2012;27(2):308-11.

64. Gazibara T, Tepavcevic DK, Svetel M, Tomic A, Stankovic I, Kostic VS, et al. Recurrent falls in Parkinson's disease after one year of follow-up: a nested case-control study. Arch Gerontol Geriatr. 2016;65:17-24.

\section{Publisher's Note}

Springer Nature remains neutral with regard to jurisdictional claims in published maps and institutional affiliations.
Ready to submit your research? Choose BMC and benefit from:

- fast, convenient online submission

- thorough peer review by experienced researchers in your field

- rapid publication on acceptance

- support for research data, including large and complex data types

- gold Open Access which fosters wider collaboration and increased citations

- maximum visibility for your research: over $100 \mathrm{M}$ website views per year

At BMC, research is always in progress.

Learn more biomedcentral.com/submissions 